\title{
Barrier model in muon implantation and application to $\mathrm{Lu}_{2} \mathrm{O}_{3}$
}

\author{
R. C. Vilão, ${ }^{1, *}$ R. B. L. Vieira, ${ }^{1,2}$ H. V. Alberto, ${ }^{1}$ J. M. Gil, ${ }^{1}$ A. Weidinger,${ }^{3}$ R. L. Lichti, ${ }^{4}$ \\ P. W. Mengyan, ${ }^{4,5}$ B. B. Baker, ${ }^{6}$ and J. S. Lord ${ }^{7}$ \\ ${ }^{1}$ CFisUC, Department of Physics, University of Coimbra, P-3004-516 Coimbra, Portugal \\ ${ }^{2}$ CICECO - Aveiro Institute of Materials, Department of Chemistry, University of Aveiro, 3810-193, Aveiro, Portugal \\ ${ }^{3}$ Helmholtz-Zentrum Berlin für Materialien und Energie, 14109 Berlin, Germany \\ ${ }^{4}$ Department of Physics, Texas Tech University, Lubbock, Texas 79409-1051, USA \\ ${ }^{5}$ Department of Physics, Northern Michigan University, Marquette, Michigan 49855, USA \\ ${ }^{6}$ Department of Physics and Engineering, Francis Marion University, Florence, South Carolina 29502, USA \\ ${ }^{7}$ ISIS Facility, Rutherford Appleton Laboratory, Chilton, Didcot, Oxon OX11 0QX, United Kingdom
}

(Received 9 June 2018; revised manuscript received 31 July 2018; published 11 September 2018)

\begin{abstract}
In implantation experiments, the implanted particle is shot with a certain energy into the material and comes to rest at a site which may not correspond to the final position. The rearrangements of the surrounding atoms to accommodate the particle, i.e., the reaction with the host atoms may require some time and lead to delayed formation of the final states. In the case of the implantation of positive muons, this rearrangement process can be followed on a timescale of nanoseconds to microseconds. A delay is expected if an energy barrier inhibits the prompt reaction. We note that the barrier height may change during the rearrangement of the lattice, thus giving rise to a two-dimensional potential profile for the conversion process. The barrier model describes the reaction path of the muon in analogy to the passage over a mountain with a saddle point. The passing over the saddle point corresponds to the lowest energy trajectory. As an example, we discuss the application of the barrier model to solid $\mathrm{Lu}_{2} \mathrm{O}_{3}$.
\end{abstract}

DOI: 10.1103/PhysRevB.98.115201

\section{INTRODUCTION}

Muon spin rotation $(\mu \mathrm{SR})$ is a widely used method in materials science [1,2]. The muons are implanted into the material, come to rest at an interstitial site, and finally form characteristic states which are distinguishable in the $\mu \mathrm{SR}$ experiment. These states are [3-6]: (i) Almost free neutral muonium $\left(\mu^{+} e^{-}\right)$at an open interstitial site. In the literature this state is called normal muonium, interstitial muonium, or atomlike or atomic muonium. Its hyperfine interaction is isotropic and close to the vacuum value. (ii) Bound neutral muonium $\left(\mu^{+} e^{-}\right)$, where muonium is incorporated into the lattice structure, i.e., it reacted with the surrounding atoms. The appearance of this state may vary significantly in $\mu \mathrm{SR}$ : In the classical $\mathrm{Si}$ case, muonium is embedded into the bond between two $\mathrm{Si}$ atoms (bond-centered muonium) and shows intermediate properties concerning electron binding energy and hyperfine interaction [3,4]. Another example of bound muonium is the shallow donor state in $\mathrm{CdS}$ and $\mathrm{ZnO}[7,8]$. (iii) Positively charged configuration $\left(\mu^{+}\right)$which in semiconductors is also in a bound configuration corresponding to the ionized version of (ii). The bound structure results from a reaction of muonium or muon with the surrounding atoms in the host.

The formation probabilities of these different states show a large variety as a function of temperature and other external parameters (e.g., electrical field) and depend strongly on the

*ruivilao@uc.pt specific material and its electronic properties, e.g., the doping $[3,4]$. Conversely, the fractions of the different $\mu$ SR signals can be used to extract properties of the material.

The formation probabilities of the different configurations are not well understood. In a number of papers delayed formation was reported [9-16]. The usual interpretation in the past was that it relates to delayed capture or loss of an electron by the muon center. We showed in a recent paper [17] that in the case of zirconia the delay is caused by the lattice rearrangement necessary to accommodate muonium. We proposed the following process [17]: Muons which have picked up an electron during the slowing down process come to rest at an interstitial site as compact atomic muonium with a large hyperfine interaction. However, this electronic configuration is not stable and the electron spreads out forming a shallow donorlike state with a weak and fluctuating hyperfine interaction. This state is observed as a fast relaxing diamagneticlike signal in $\mu \mathrm{SR}$. The lifetime (ns to $\mu \mathrm{s}$ ) ends when the ground state is formed.

In the present paper we describe this process in analogy to traversing a mountain from one side to the other (atomic to bound). A barrier exists if the formation energy along the transition trajectory goes through a maximum. We apply the barrier model to the recently measured $\mathrm{Lu}_{2} \mathrm{O}_{3}$ data.

The model is not applicable to cases in which the ground state is formed promptly without going through the transition state. Examples are possibly Si and Ge. The formation of the transition state is suppressed, e.g., if the bandlike electron configuration (shallow donor state) has higher energy than the atomic state, both in the unrelaxed lattice. The branching 
between the bandlike and atomic configuration depends on the material but also on temperature if thermal excitation is needed to form the bandlike state. Thus, the delayed formation of the final states may have different reasons, either rearrangement of the lattice or interaction with charge carriers. In the present paper, we discuss the phenomena related to the lattice rearrangement necessary to accommodate the implanted muon.

In the past few years we have used muon spin spectroscopy in order to model the behavior of the isolated hydrogen impurity in dielectric oxides [13,18-23]. These materials find increasing interest and application in modern electronic devices [24-26]. The goal of the muon spin spectroscopy experiments is to obtain a microscopic understanding of these materials on an atomistic scale, in particular regarding the characterization of the hydrogen impurity, which is adequately modeled by the muonium analog $[2,4,5]$. This work has been performed often in conjunction with ab initio DFT calculations on the same systems [27-31]. Many similarities have been found, and the typical signature of the acceptor configuration shows up as the almost free muonium in an interstitial site $[5,6,13,18,20,28,31-33]$, whereas the donor configuration typically appears as a shallow-donor state bound to oxygen $[5-8,20,23]$. However, significant unexplained differences have been found in particular with respect to the formation probabilities of the different states.

Among rare-earth oxides, $\mathrm{Lu}_{2} \mathrm{O}_{3}$ is particularly relevant due to its chemical and mechanical stability [34,35], as well as due to its thermal, optical, and electric properties [35-38]. It has attracted much interest as host material for continuous wave laser operations [35] and is a promising potential gate material for the next generation of field-effect transistors in high- $\kappa$ technology [26]. However, little is known about the defect physics of $\mathrm{Lu}_{2} \mathrm{O}_{3}$. The relevant hydrogen impurity has been addressed recently by da Silva et al. [39], where a thorough theoretical approach has been complemented by the identification of the formation of an atomlike muonium configuration. We now present an extended report of this investigation and analyze the data in the barrier model developed in this paper.

\section{EXPERIMENTAL DETAILS AND RESULTS}

A polycrystalline $\mathrm{Lu}_{2} \mathrm{O}_{3}$ sample used in the present study was obtained commercially from Alfa-Aesar (REActon 99.995\%). The $\mu$ SR measurements were performed partially at TRIUMF (Vancouver, Canada) [40] and partially at ISIS (UK) [41]. At TRIUMF, high-transverse field (up to $7 \mathrm{~T}$ ) measurements were performed at the HiTime spectrometer on the M15 surface muon channel, and zero-field measurements at the LAMPF spectrometer on the M20 muon channel. At ISIS, low transverse field $(10 \mathrm{mT})$ and longitudinal field data were obtained with the EMU spectrometer in the temperature range from 8 to $700 \mathrm{~K}$. Each of these experiments revealed particular aspects of this investigation (see experimental results). Details of the instruments and of the $\mu \mathrm{SR}$ method can be found on the web pages of TRIUMF and ISIS [40,41].

Figure 1 shows the Fourier spectrum of a high-field $\mu$ SR measurement at $T=300 \mathrm{~K}$. Clearly the lines of diamagnetic muon $v_{d}$ and the lower-frequency line $v_{12}$ of atomlike

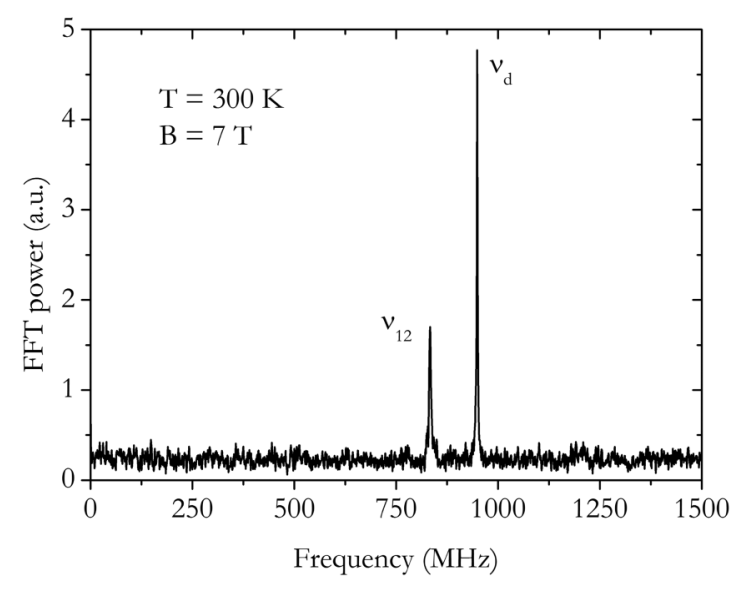

FIG. 1. Fast Fourier transform of the $\mu \mathrm{SR}$ time spectrum at $T=$ $300 \mathrm{~K}$ for applied transverse field of $7 \mathrm{~T}$, showing the presence of the diamagnetic frequency $v_{d}$ and the muonium $v_{12}$ frequency.

interstitial muonium are seen. The higher-frequency line $v_{34}$ of muonium is not accessible in this experiment.[39]

At low temperatures, an additional fast relaxing signal is observed which is best seen in the longitudinal field measurement (Fig. 2).

Thus, all together, three signals can be distinguished: The muonium signal, the diamagnetic signal, and the fast relaxing signal. The fractions vary with temperature (Fig. 3). At low temperatures $(T<50 \mathrm{~K})$, the diamagnetic fraction accounts for about $30 \%$ of all muons, the fast fraction and the muonium fraction each for about $10 \%$. Thus, about $50 \%$ of the muons show no signal here (missing fraction). At $T=300 \mathrm{~K}$, atomic muonium is the dominant species with a fraction well over $40 \%$, the diamagnetic signal has about $25 \%$ fraction, and the fast signal does not exist at this temperature.

There is an overall missing fraction of about $30 \%$ to $50 \%$ which is due to depolarization by a precursor process. A further missing fraction in the temperature range between

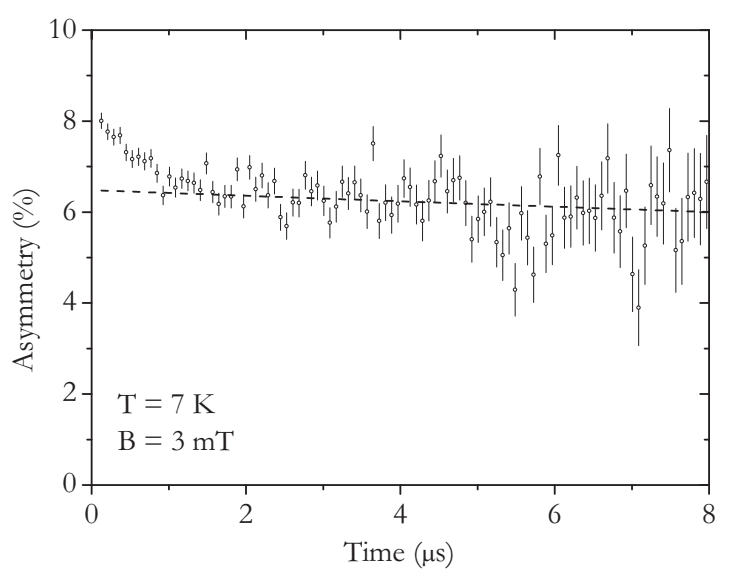

FIG. 2. Longitudinal-field $\mu \mathrm{SR}$ spectrum at $B_{\mathrm{LF}}=3 \mathrm{mT}$ and $T=7 \mathrm{~K}$. The dotted line corresponds to a fit to the long-time behavior; it gives a relaxation rate of 0.010 (4) $\mu \mathrm{s}^{-1}$. Clearly a fast relaxing component (relaxation rate approximately $6 \mu \mathrm{s}^{-1}$ ) is seen at the beginning of the time spectrum. 


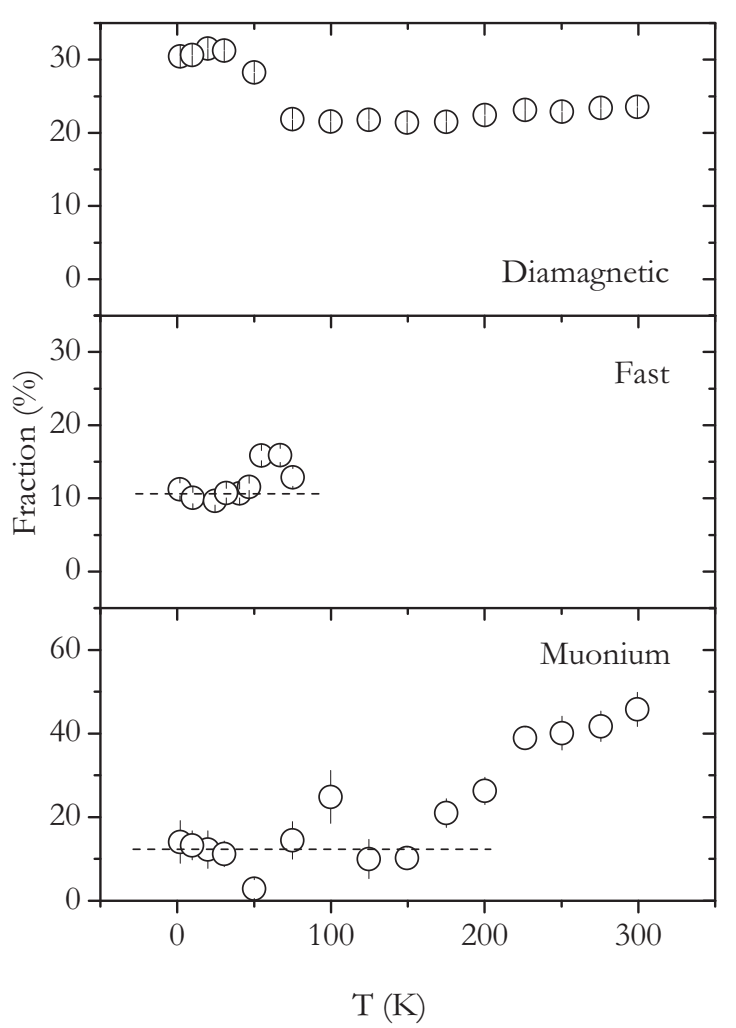

FIG. 3. Temperature dependence of the three muon fractions in $\mathrm{Lu}_{2} \mathrm{O}_{3}$ as a function of temperature (diamagnetic and fast from zerofield measurement, muonium from $7 \mathrm{~T}$ experiment). For muonium, the fraction of the observed lower muonium line was multiplied by two to account for the nonmeasured upper muonium line.

about 50 and $200 \mathrm{~K}$ is explained by dephasing during a specific conversion (see Sec. IV B below).

\section{A. The muonium state}

The atomlike muonium state (Fig. 1) is observed in $\mathrm{Lu}_{2} \mathrm{O}_{3}$ at all temperatures in the range of the present high-field experiment ( 8 to $300 \mathrm{~K}$ ). The data have been analyzed with a superposition of cosine functions as in Ref. [39], $\cos (\omega t+\phi)$, where $\omega$ is the angular frequency and $\phi$ is a phase. The phase has a constant contribution from the geometrical arrangement of the detectors (here approximately $100 \mathrm{deg}$ ) and a possible shift due to a precursor effect.

The hyperfine interaction extrapolated to zero temperature is 3629 (2) MHz, corresponding to about $81 \%$ of the vacuum value; it decreases slightly with temperature due to vibrations of the surrounding atoms. The fit curve is obtained assuming an Einstein model for the vibrations with an activation energy of 21(2) meV [32].

The fraction of atomic muonium (second frame of Fig. 4) amounts to over $40 \%$ around room temperature but decreases with decreasing temperature. Below about $200 \mathrm{~K}$, the analysis of the time spectrum clearly reveals a signal at the muonium frequency with an amplitude corresponding to about $10 \%$ fraction.

The phase of the atomic muonium signal (third frame of Fig. 4) is constant within errors and is consistent with no

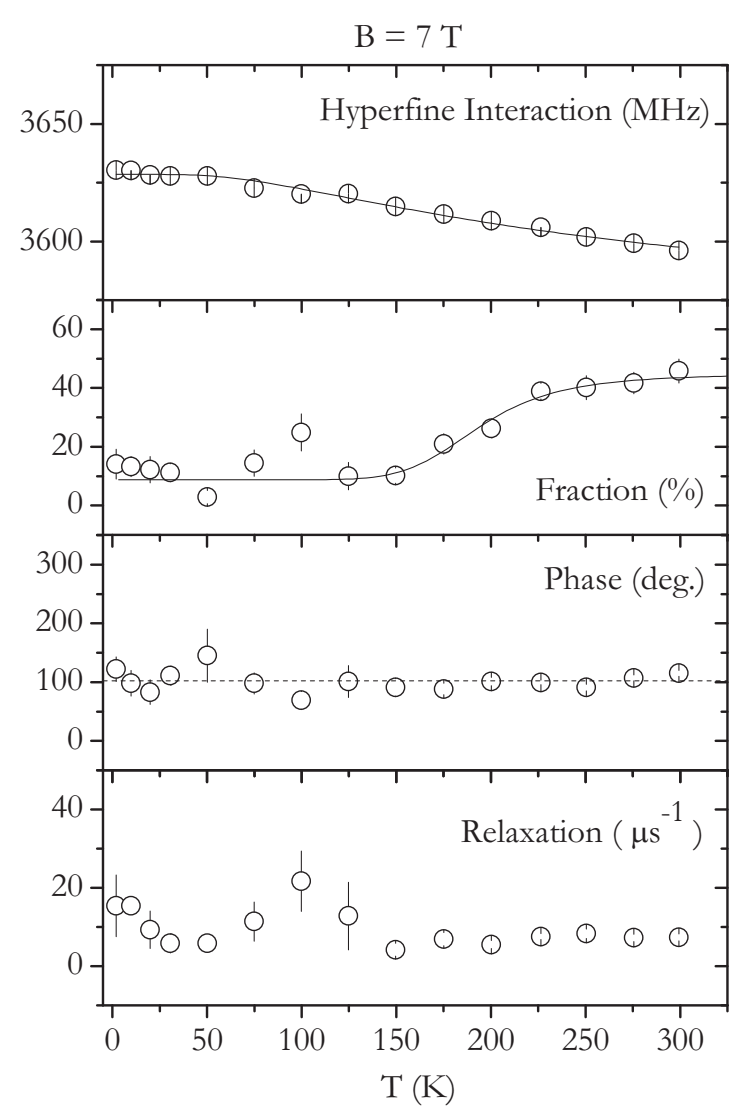

FIG. 4. Temperature dependence of the parameters of atomic muonium in $\mathrm{Lu}_{2} \mathrm{O}_{3}$ at an external magnetic field of $B=7 \mathrm{~T}$. The solid lines in the upper two frames are fits with the Einstein model and an Arrhenius function, respectively. The phase in the third frame is the phase of the $v_{12}$ frequency $(833 \mathrm{MHz})$, the dashed line corresponds to the phase expected for this frequency from a calibration curve using silver.

phase shift. A phase shift is expected for a single step reaction but it averages to zero for repeated forth and back reactions. The latter is apparently the case in the present situation. An important consequence of this result is that the reduction of the fraction at lower temperatures is not due to dephasing (delayed formation from a diamagneticlike precursor) but corresponds to a lower formation probability of atomic muonium.

The rather large and fairly constant relaxation rate is probably due to slight variations of the local internal field at the muon site. Large relaxations of atomic muonium signals are commonly reported in the literature $[4,32]$.

\section{B. The fast relaxing signal}

The time spectrum in Fig. 2 clearly reveals a fast relaxing signal in $\mathrm{Lu}_{2} \mathrm{O}_{3}$. The properties of this signal as obtained from zero-field measurements are displayed in Fig. 5. Below about $50 \mathrm{~K}$, the fraction amounts to approximately $10 \%$ and the relaxation rate is on the order of $6 \mu \mathrm{s}^{-1}$. Above $50 \mathrm{~K}$, the signal becomes weaker and a clear separation between fraction and relaxation effects becomes difficult. However, in spite of this difficulty, the data indicate that the relaxation rate increases above $50 \mathrm{~K}$. We interpret this behavior as 


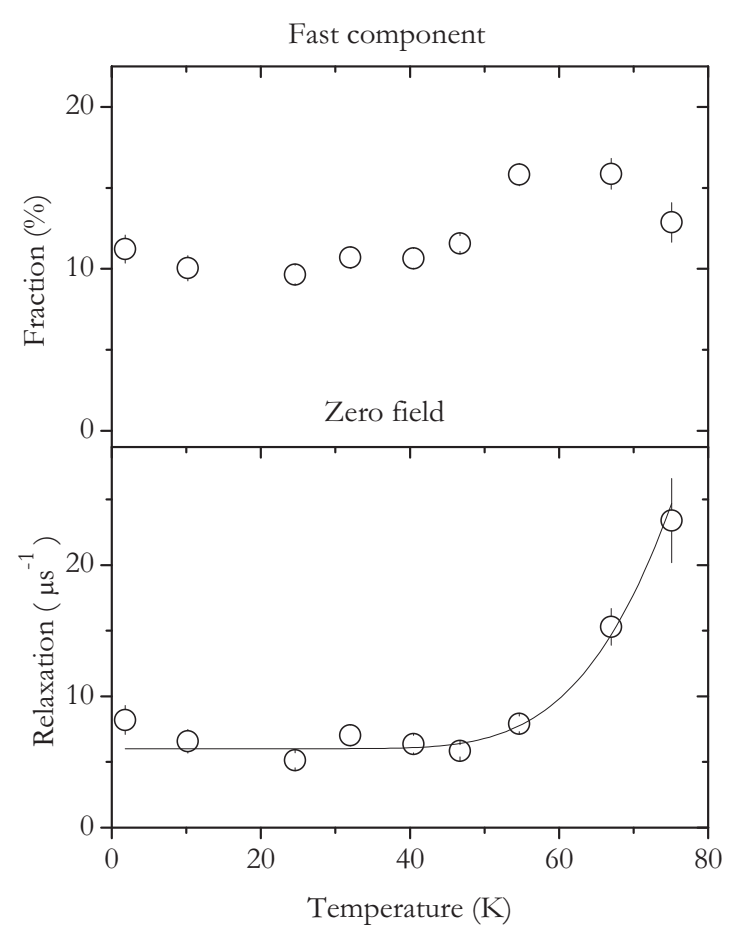

FIG. 5. Fraction and relaxation rate of the fast relaxing signal as a function of temperature.

a conversion of a precursor state to atomic muonium (see discussion below).

\section{The diamagnetic signal}

The diamagnetic signal from the low transverse field is shown in Fig. 6. The slight difference in the absolute values compared to the zero-field experiment shown in Fig. 3 is likely due a normalization effect, in particular with respect to the zero-field background. This does not affect the overall shape of the temperature dependence. The diamagnetic signal has different origins. Part of it is due to incoming $\mu^{+}$ which has not picked up an electron in the charge exchange process during slowing down. The other part stems from neutral muonium which was formed in the charge exchange process but has converted to a diamagneticlike configuration. We distinguish two possibilities; either the electron remains near the muon but is in a transition state [17] with a weak hyperfine interaction which fluctuates and averages to almost zero, or the electron is lost during the reaction of muonium with the lattice and the $\mu^{+}$state is observed. Experimentally, we cannot distinguish between these two possibilities in the present case.

The diamagnetic fraction (upper frame of Fig. 6) shows a two-step recovery with increasing temperature. A fit with Boltzmann functions [13] (solid line) yields activation energies of 0.07 and $0.7 \mathrm{eV}$, respectively.

At low temperatures (below about $60 \mathrm{~K}$ ), a remarkable increase of the fraction with decreasing temperature is observed. This is not due to a conversion to muonium, because that would imply an increase of the relaxation of the diamagnetic component, and this effect is not observed. Also, the interpretation of the decrease with increasing temperature as due to

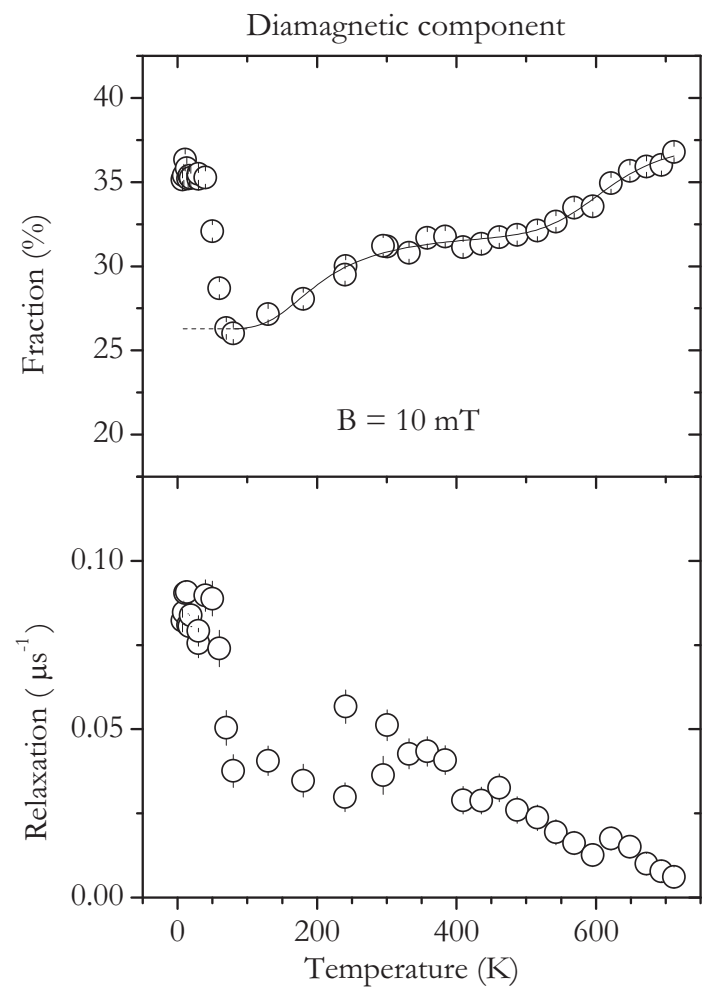

FIG. 6. Fraction and relaxation rate of the diamagnetic signal in $\mathrm{Lu}_{2} \mathrm{O}_{3}$ at $B=10 \mathrm{mT}$. The signal may partially be due to a very weakly relaxing paramagnetic component with a diamagneticlike frequency behavior. The solid line in the fraction plot corresponds to a two-step recovery with activation energies of $0.07(2)$ and $0.7(3)$ $\mathrm{eV}$, respectively. The low-temperature peak in the fraction plot is attributed to a "hot-atom" effect (see section "thermal spike" below). The different values of the relaxation at around $300 \mathrm{~K}$ were obtained after changes in the experimental setup; they indicate systematic uncertainties.

dephasing is not realistic, since for very similar frequencies the dephasing effect is negligible. Therefore, we attribute this anomaly to a "hot atom" effect and discuss it below in the concept of a "thermal spike" [42].

The depolarization rate $\sigma$ of the diamagneticlike fraction (lower frame of Fig. 6) amounts at low temperatures to about $0.08 \mu \mathrm{s}^{-1}$. This value is at the upper end of the expected depolarization rate due to nuclear moments (the calculated depolarization value depends on the position of the muon with respect to the surrounding atoms; it is not well known). Thus the signal may possibly correspond to a pure diamagnetic configuration. However, the shape of the temperature dependence (rather abrupt change of the depolarization rate to about half of its value around $60 \mathrm{~K}$ ) is better understood if one assumes that part of the depolarization is due to a very weak paramagnetic state which disappears above $60 \mathrm{~K}$. The gradual decrease of the depolarization rate above room temperature is attributed to long range diffusion.

\section{Decoupling experiment}

The decoupling measurement was performed in longitudinal field geometry at the temperature of $7 \mathrm{~K}$. A typical $\mu \mathrm{SR}$ 


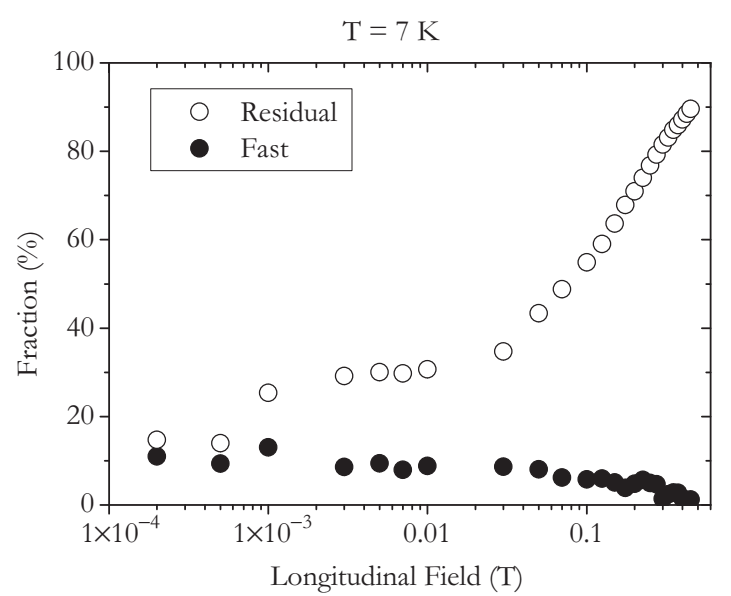

FIG. 7. Decoupling experiment at $T=7 \mathrm{~K}$. The residual polarization (open circles) shows a recovery in the field range around $100 \mathrm{mT}$; it corresponds to the decoupling of an interaction with atomic muonium characteristics but it cannot be fitted by a unique hyperfine interaction. At about $1 \mathrm{mT}$ a low interaction is decoupled corresponding probably to a weak paramagnetic component (see text). The fast relaxing component (filled circles) is not decoupled at low fields, but the signal disappears when atomic muonium is decoupled.

time spectrum is shown in Fig. 2: A very slowly relaxing (almost constant) signal is superimposed by a fast relaxing signal at short times. At fields below $1 \mathrm{mT}$ an additional slowly relaxing signal is observed (relaxation rate approximately $0.1 \mu \mathrm{s}^{-1}$ ) but is not shown here. The fraction of the almost constant signal (attributed to the residual polarization part) and the fraction of the fast relaxing signal are displayed in Fig. 7.

The main decoupling occurs at rather high fields around $100 \mathrm{mT}$. This indicates that the strong atomic-muonium-like interaction is decoupled in this region. However, the shape of the curve cannot be fitted assuming a decoupling of the $3.6 \mathrm{GHz}$ muonium observed directly in the high transverse field experiment. We suppose that several components, including fluctuating ones, are contained in this curve.

A slowly relaxing component, probably a paramagnetic state with a very small hyperfine interaction, becomes decoupled at fields below $1 \mathrm{mT}$ (increase of the decoupled fraction in this region). In the transverse field experiment this component cannot be distinguished from the real diamagnetic fraction because of too similar parameters. Thus, not much is known about this component.

The fast component remains constant up to fields of several $10 \mathrm{mT}$. This excludes that this component is due to an anisotropy of the muonium state discussed before. The disappearance of the fast signal coincides with the decoupling of the atomlike muonium interaction. This indicates that the fast signal corresponds to a precursor state (transition state) which converts to atomic muonium.

\section{THE BARRIER MODEL}

The barrier model describes the conversion of incoming muonium $\left(\mu^{+} e^{-}\right)$to one of the three final configurations:

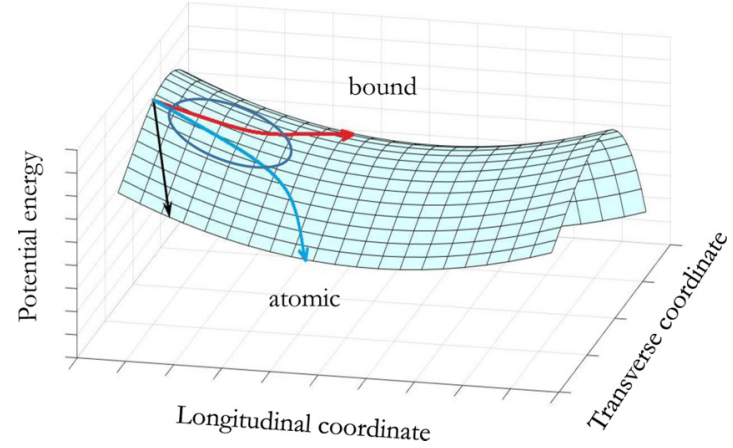

FIG. 8. Muonium trajectories (black and colored lines) on a two-dimensional potential landscape during the relaxation of the lattice. Reaction coordinates parallel (longitudinal) and perpendicular (transverse) to the ridge separating the atomic configuration from the bound configuration are indicated. (Note: Muonium has additionally internal coordinates corresponding to the distance of muon and electron.) Different potential profiles reflect the changes of the atomic arrangement during the lattice relaxation. The red line shows the path over the barrier (formation of a bound configuration). The black line and the blue line correspond to the prompt and the delayed formation of atomlike muonium, respectively. The encircled region indicates the area of the fairly long-lived (ns to $\mu \mathrm{s}$ ) transition state in which the electron is only weakly bound, resulting in a small hyperfine interaction.

Atomlike muonium, bound muonium, and bound muon. As an analog, one may have in mind the crossing of a mountain from one side to the other through a saddle point. The barrier model is widely used in chemistry to describe chemical reactions by calculating trajectories on potential energy surfaces [43]. In the present case, the formation of the final muon states may be considered as a reaction of the muon with the surrounding atoms.

Figure 8 shows schematically a two-dimensional potential profile with a saddle point. In the analog mentioned above, the two dimensions correspond, e.g., to the north-south and the east-west direction. For muonium, one dimension (transverse) corresponds to the coordinate perpendicular to the ridge separating the atomic configuration from the bound configuration. The other coordinate (longitudinal) is parallel to this ridge. It corresponds also to a timescale: Muonium proceeds on the slope of the mountain; the barrier height changes due to lattice relaxation. The different potential profiles refer to actual atomic configurations of the relaxing lattice. The lowest energy path of muonium to a bound configuration (muonium or $\mu^{+}$) goes over the saddle point. The right part of the figure is of no significance, it just indicates that there is no lower potential than the saddle point potential. The trajectories start at a point high up in the mountain; muonium is brought there by the implantation (see also the paragraph "starting point" below).

We consider here only those muons which have picked up an electron in the charge exchange process and stop as neutral muonium. The muons stopping directly in the positively charged configuration $\mu^{+}$are expected to react immediately with the lattice and form a bound state. Thus, a diamagnetic fraction in addition to the fractions originating from muonium may exist. 
Starting point. The stopped muonium has initially additional potential energy since it is embedded in the unrelaxed lattice. The value of this energy is in the order of $1 \mathrm{eV}$ [44] and depends on the host material. Thus (see Fig. 8), muonium may start from a potential above the saddle point but still on the atomic muonium side of the barrier. There are other situations possible for the starting point, but the one indicated in Fig. 8 is probably realistic for our example case.

Weakly bound state. The pickup of the electron in the charge exchange process occurs, for cross section reasons, into an atomic level of muonium. However, this state may not be stable for longer times and decay into a shallow-donorlike electron configuration. Very generally, the shallow donor state configuration is the expected configuration if a positive charge is embedded in a dielectric medium with effectivemass conduction band structure. We note that the formation of the weakly bound donor state may need a thermal activation if the initial atomic level does not lie in the conduction band of the host material.

Barrier height. Muonium loses potential energy by excitation of local vibrational modes via electron-phonon coupling. At the same time, the lattice configuration around muonium changes due to thermal fluctuations (partially due to the emitted phonons). Thus, trajectories as indicated by the black and colored lines in Fig. 1 may occur.

Three basic types of trajectories may be distinguished (see Fig. 8): (i) Prompt descent on the atomic muonium side of the barrier (black line), (ii) delayed descent on the muonium side (blue line), and (iii) path over the barrier (red line). The first two trajectories lead to the formation of atomlike muonium (prompt or delayed), whereas the third path corresponds to the formation of a bound configuration. The barrier height determines, together with temperature and other parameters, the branching between atomlike and bound muonium.

Ionization. In the transition state, the electron is only weakly bound to the muon and the binding energy fluctuates with changing lattice configuration. Thus, it is possible that along the trajectory the muonium electron gets lost to the conduction band or to traps and the positive muon remains behind. It then reacts immediately with the lattice and forms a bound configuration. Ionization determines the branching between bare muon and muonium configurations.

\section{APPLICATION OF THE BARRIER MODEL TO $\mathrm{Lu}_{2} \mathrm{O}_{3}$}

In $\mathrm{Lu}_{2} \mathrm{O}_{3}$, atomlike muonium is more stable than bound muonium [39]. This favors the descent on the atomic side of the potential profile, i.e., the formation of atomlike muonium. In Fig. 8, two atomic-side descents are indicated, one prompt (black) and one delayed (blue).

\section{A. Prompt formation of atomic muonium (black line in Fig. 8)}

The immediate descent on the atomic side of the potential profile corresponds to the direct conversion of initial muonium to the final configuration in the relaxed lattice. We assign the atomic muonium presented in Figs. 1 and 3 to this process. At $300 \mathrm{~K}$, the prompt process accounts for about $46 \%$ of all muons (the visible line has about $23 \%$ fraction). At low temperatures, the visible fraction of atomic muonium goes down (see Fig. 4). This could be due either to a reduced formation probability or to dephasing caused by delayed formation from a precursor with a different frequency. The fact that no appreciable phase shift is observed for the remaining atomic fraction below $200 \mathrm{~K}$ points in the direction that this part is formed promptly and not via a transition state. Fast electron spin fluctuations which could suppress the phase shift but not the amplitude are unlikely at this low temperature. The signal is very weak and no detailed information is available for it. Therefore no definite assignment can be made, but prompt conversion is plausible.

\section{B. Delayed formation of atomic muonium (blue line in Fig. 8)}

A fraction of stopped muonia forms quickly the weakly bound state (with diamagneticlike frequency) and remains in this state until it decays to relaxed atomic muonium. Thus, there is a conversion from the initial diamagneticlike signal to the final muonium signal with a mean conversion time $\tau$. We assign the fast relaxing signal presented in Fig. 5 to the initial state in this conversion process.

The main parameter for this conversion is given by (for $\mathrm{Lu}_{2} \mathrm{O}_{3}$ with $A=3.63 \mathrm{GHz}$; high field limit, $7 \mathrm{~T}$ data):

$$
\Delta \omega \approx \pi A=11.4 \mathrm{~ns}^{-1},
$$

where $\Delta \omega$ is the difference of the angular frequency of the muonium line and the diamagnetic line. The relaxation of the initial state (fast relaxing signal) and the fraction of the final muonium state are correlated by

$$
\begin{aligned}
& \lambda_{\text {fast }}=\lambda_{0}+\frac{1}{\tau}, \\
& f_{\mathrm{Mu}}=\frac{1}{\sqrt{1+(\Delta \omega \tau)^{2}}},
\end{aligned}
$$

where $\lambda_{0}$ describes an offset due, e.g., to line broadening by a fluctuating hyperfine interaction and $1 / \tau$ is the relaxation due to the finite lifetime of the state. The second part of Eq. (2) describes the amplitude reduction in the high field limit [see Eq. (1)] [32]. At lower fields, the detailed structure of muonium has to be taken into account [4]. We do not make use of the exact $\Delta \omega$ values in the analysis of the low field data. If the mean conversion time is longer than about $1 \mathrm{~ns}$ ( $\tau>1 \mathrm{~ns}$ ), then dephasing in transverse field is complete [see Eqs. (1) and (2)] and no muonium signal is observed from this process. This explains the low muonium fraction at low temperatures. The still observed small fraction at low temperatures is attributed to the branching between prompt and delayed formation of atomic muonium.

The delayed muonium formation via the transition state is characterized (see Fig. 9) by (i) the disappearance of the fast signal (initial state) through the increase of the relaxation rate (lifetime shortening) around $70 \mathrm{~K}$ and by (ii) the appearance of the muonium signal (final state) at around $200 \mathrm{~K}$ when the transition is so fast that no dephasing takes place. In the intermediate temperature region, no signal from this process is seen due to dephasing.

In principle, one could interpolate between these two regions, e.g., by assuming an Arrhenius behavior for the lifetime $\tau$ of the initial state. However, this is not very meaningful since first of all the lifetime shortening with temperature is 


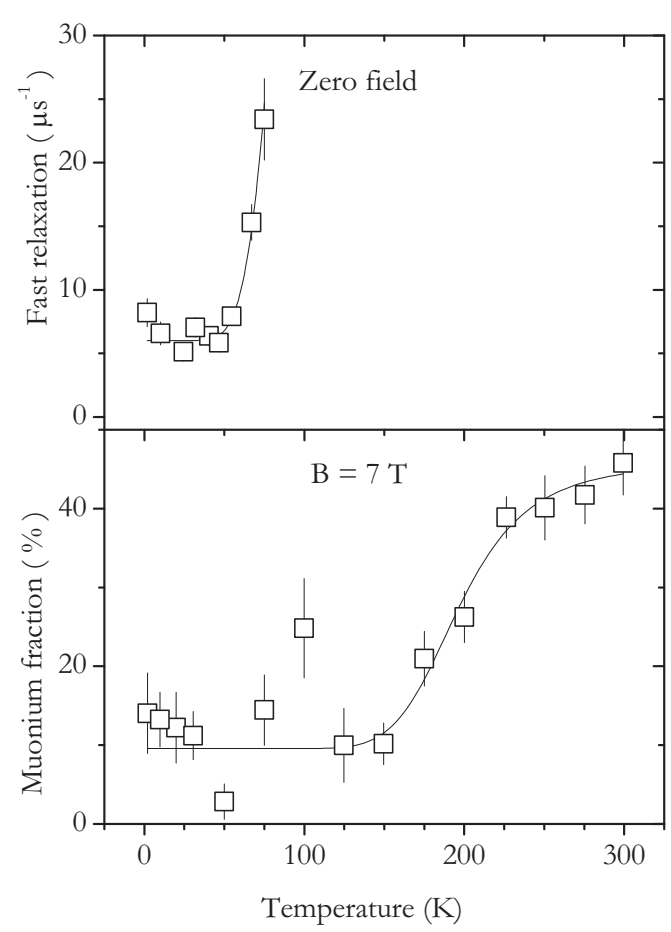

FIG. 9. Fast relaxation rate (from zero-field measurement) and muonium fraction (from $7 \mathrm{~T}$ measurement) as function of temperature. The lines in the figure are guides to the eye.

not necessarily Arrhenius-like and second as the lifetime gets very short, the intermediate weakly bound state may not be formed at all and a direct conversion to atomic muonium may occur. Therefore, the solid lines in Fig. 9, though obtained with Arrhenius functions, are considered as guides to the eye.

\section{Formation of a bound configuration (red line in Fig. 8)}

The diamagnetic signal (Fig. 6) is assigned, at least partially (some part may be due to muons which stopped directly as $\mu^{+}$), to the conversion of the originally stopped muonium to a bound configuration. During this conversion path (red line in Fig. 8) the electron may get lost (ionization) and a change of the weakly paramagnetic to a purely diamagnetic state may take place. In the present experiment, we cannot distinguish between the signals of these two configurations and therefore present the data for the sum of these two components.

Thermal activation over the conversion barrier is assumed. The black line in Fig. 6 represents a two-step recovery with activation energies of 0.07 and $0.7 \mathrm{eV}$, respectively. The recovery in two steps with very different activation energies seems to indicate that one conversion occurs from the ground state and the other one from an excited level. Such an excited configuration is the transition state for which the conversion over the barrier requires only a small activation. We tentatively assign the $0.7 \mathrm{eV}$ activation to the conversion from the ground state atomic configuration and the much smaller activation $(0.07 \mathrm{eV})$ to the conversion from the transition state, i.e., to a jump over the barrier while the muonium is still in the high potential area. This can qualitatively be seen on the potential picture in Fig. 8.
Thermal spike. As mentioned before, the re-increase of the diamagnetic fraction with decreasing temperature below about $60 \mathrm{~K}$ (see Fig. 6) is attributed to a "hot atom" effect [45] which may be qualitatively understood in the following way: muonium stopped in the unrelaxed lattice is at a higher potential than ground state muonium and therefore is more reactive ("hot") than the latter. The extra potential energy is gradually converted into local heat which spreads out into the surrounding. One may thus speak of a thermal (or temperature) spike which decays with time [42]. At low temperatures, the local heat remains relatively long at the muon site (because of low thermal conductivity) but decreases rapidly as the temperature increases. No low-temperature thermal conductivity values are available for the present substance but it is known that the thermal conductivity of nonmetals increases very strongly with temperature from a very low value at low temperatures $[46,47]$. Thus the thermal spike effect disappears at higher temperatures, here above about $60 \mathrm{~K}$, due to rapid heat flow into the surroundings.

A more detailed discussion of this effect is beyond the scope of the present paper. We would, however, like to mention that this low temperature effect may be present also in other muon experiments and deserves further attention.

\section{CONCLUSIONS}

The barrier model assumes that the originally formed muonium (electron pickup in the charge exchange circle) is hindered by a barrier to react with the host lattice. However, thermal activation may initiate the reaction. The end products are almost free muonium at an interstitial site or muonium (or bare muon if the electron is lost during the conversion) embedded in the lattice structure of the host. We note that, as a local probe, the muon is not very sensitive to defects and other global properties of the sample [13]. Since the implantation places the muon statistically into the sample, it sees mainly the intrinsic surroundings in the lattice. The sample used here is of high purity (see experimental details), so that we can assume that we are measuring intrinsic properties. The proposed model is therefore of significance beyond the particular sample and material presented here.

Since the barrier height depends on the actual atomic configuration (which changes with time during the lattice relaxation), the potential profile for the reaction (Fig. 8) presents a two-dimensional character. The waiting for a favorable potential profile (in particular the saddle point configuration) competes with the potential loss of muonium due to the spread out of the local energy originating from implantation.

This leads to the following assignment of the observed signals:

(i) Atomic muonium, for which the hyperfine lines are observed, corresponds to the prompt formation of this state from the incoming compact muonium without going through the transition state (prompt descent on the muonium side of the potential profile in Fig. 8).

(ii) The fast relaxing signal is assigned to the relatively long-lived weakly bound state (formed from the incoming muon with an electron from the charge exchange cycle), still in the basically unrelaxed lattice. The lifetime of the transition 
state ends by conversion to relaxed atomic muonium (delayed descent on the atomic side of the barrier in Fig. 8).

(iii) The diamagnetic signal has various origins: Part of the signal is due to incoming $\mu^{+}$which, in oxides, forms promptly a bound configuration. The other part stems from muonium surmounting the potential barrier and forming a bound configuration. During this transition, the electron may get lost and the final state corresponds actually to bare muon. But it is also possible that the electron remains bound but the final bound muonium state is not observed due to late formation.

We showed on the example $\mathrm{Lu}_{2} \mathrm{O}_{3}$ that the barrier model can explain the rich variety of experimental findings of this experiment. The model is applicable also to other similar systems and will shed light on the so far badly understood final step in muon implantation. As in chemistry [43], formation rates and reaction trajectories can be calculated if the potential energy surface is known. Theoretical calculations in this direction are scarce [27,30]. At present, it is more likely that one chooses the opposite way and infers barrier heights and reaction trajectories from experiment findings.

\section{ACKNOWLEDGMENTS}

The use of the ISIS and TRIUMF beams and the help of the respective muon teams are gratefully acknowledged. This work was supported with funds from (i) FEDER (Programa Operacional Factores de Competitividade COMPETE) and from FCT - Fundação para a Ciência e a Tecnologia under projects UID/FIS/04564/2016 and PTDC/FIS/102722/2008; (ii) Ph.D. grant SFRH/BD/87343/2012 from FCT - Fundação para a Ciência e a Tecnologia (RBLV). The Texas Tech group gratefully acknowledges support from the Texas Research Incentive Program.
[1] P. J. C. King, R. de Renzi, S. P. Cottrell, A. D. Hillier, and S. F. J. Cox, ISIS muons for materials and molecular science studies, Phys. Scr. 88, 068502 (2013).

[2] S. F. J. Cox, R. L. Lichti, J. S. Lord, E. A. Davis, R. C. Vilão, J. M. Gil, T. D. Veal, and Y. G. Celebi, The first 25 years of semiconductor muonics at ISIS, modelling the electrical activity of hydrogen in inorganic semiconductors and high- $\kappa$ dielectrics, Phys. Scr. 88, 068503 (2013).

[3] S. F. J. Cox, Muonium as a model for interstitial hydrogen in the semiconducting and semimetallic elements, Rep. Prog. Phys. 72, 116501 (2009).

[4] B. D. Patterson, Muonium states in semiconductors, Rev. Mod. Phys. 60, 69 (1988).

[5] A. Weidinger, J. M. Gil, H. V. Alberto, R. C. Vilão, J. Piroto Duarte, N. Ayres de Campos, and S. F. J. Cox, Shallow donor versus deep acceptor state in II-VI semiconductor compounds, Phys. B: Condens. Matter 326, 124 (2003).

[6] R. C. Vilão, J. M. Gil, A. Weidinger, H. V. Alberto, J. Piroto Duarte, N. Ayres de Campos, R. L. Lichti, K. H. Chow, and S. F. J. Cox, Information on hydrogen states in II-VI semiconductor compounds from a study of their muonium analogues, Nucl. Instrum. Methods Phys. Res., Sect. A 580, 438 (2007).

[7] J. M. Gil, H. V. Alberto, R. C. Vilão, J. P. Duarte, P. J. Mendes, L. P. Ferreira, N. Ayres de Campos, A. Weidinger, J. Krauser, Ch. Niedermayer, and S. F. J. Cox, Novel Muonium State in CdS, Phys. Rev. Lett. 83, 5294 (1999).

[8] S. F. J. Cox, E. A. Davis, S. P. Cottrell, P. J. C. King, J. S. Lord, J. M. Gil, H. V. Alberto, R. C. Vilão, J. Piroto Duarte, N. Ayres de Campos, A. Weidinger, R. L. Lichti, and S. J. C. Irvine, Experimental Confirmation of the Predicted Shallow Donor Hydrogen State in Zinc Oxide, Phys. Rev. Lett. 86, 2601 (2001).

[9] S. R. Kreitzman, R. F. Kiefl, D. R. Noakes, J. H. Brewer, and E. J. Ansaldo, Thermally activated muonium formation in $\mathrm{Al}_{2} \mathrm{O}_{3}$ and $\mathrm{BaF}_{2}$, Hyperfine Interact. 32, 521 (1986).

[10] V. Storchak, J. H. Brewer, and G. D. Morris, Quantum transport of electronic polarons in sapphire, Phys. Rev. B 56, 55 (1997).

[11] J. D. Brewer, J. H. Brewer, G. D. Morris, D. G. Eshchenko, and V. G. Storchak, Electric-field-induced muonium formation in sapphire, Phys. B: Condens. Matter 289-290, 428 (2000).
[12] I. Fan, K. H. Chow, B. Hitti, R. Scheuermann, A. I. Mansour, W. A. MacFarlane, B. E. Schultz, M. Egilmez, J. Jung, Y. G. Celebi, H. N. Bani-Salameh, B. R. Carroll, J. E. Vernon, and R. L. Lichti, Influence of photoexcitation on the diamagnetic muonium states in Ge studied via their precession signatures, Phys. Rev. B 78, 153203 (2008).

[13] R. C. Vilão, A. G. Marinopoulos, R. B. L. Vieira, A. Weidinger, H. V. Alberto, J. P. Duarte, J. M. Gil, J. S. Lord, and S. F. J. Cox, Hydrogen impurity in paratellurite $\alpha-\mathrm{TeO}_{2}$ : Muon-spin rotation and $a b$ initio studies, Phys. Rev. B 84, 045201 (2011).

[14] T. Prokscha, K. H. Chow, E. Stilp, A. Suter, H. Luetkens, E. Morenzoni, G. J. Nieuwenhuys, Z. Salman, and R. Scheuermann, Photo-induced persistent inversion of germanium in a 200-nm-deep surface region, Sci. Rep. 3, 2569 (2013).

[15] H. V. Alberto, R. C. Vilão, R. B. L. Vieira, J. M. Gil, A. Weidinger, M. G. Sousa, J. P. Teixeira, A. F. da Cunha, P. M. P. Salomé, P. A. Fernandes, T. Törndahl, T. Prokscha, A. Suter, and Z. Salman, Slow-muon study of quaternary solar-cell materials: Single layers and $p-n$ junctions, Phys. Rev. Mater. 2, 025402 (2018).

[16] H. V. Alberto, R. C. Vilão, J. M. Gil, J. Piroto Duarte, R. B. L. Vieira, A. Weidinger, J. P. Leitão, A. F. Cunha, M. G. Sousa, J. P. Teixeira, P. A. Fernandes, P. M. P. Salomé, K. Timmo, M. Loorits, A. Amato, H. Luetkens, T. Prokscha, A. Suter, and Z. Salman, Muonium states in $\mathrm{Cu}_{2} \mathrm{ZnSnS}_{4}$ solar cell material, J. Phys.: Conf. Ser. 551, 012045 (2014).

[17] R. C. Vilão, R. B. L. Vieira, H. V. Alberto, J. M. Gil, and A. Weidinger, Role of the transition state in muon implantation, Phys. Rev. B 96, 195205 (2017).

[18] R. B. L. Vieira, R. C. Vilão, A. G. Marinopoulos, P. M. Gordo, J. A. Paixão, H. V. Alberto, J. M. Gil, A. Weidinger, R. L. Lichti, B. Baker, P. W. Mengyan, and J. S. Lord, Isolated hydrogen configurations in zirconia as seen by muon spin spectroscopy and $a b$ initio calculations, Phys. Rev. B 94, 115207 (2016).

[19] R. C. Vilão, R. B. L. Vieira, H. V. Alberto, J. M. Gil, A. Weidinger, R. L. Lichti, B. B. Baker, P. W. Mengyan, and J. $\mathrm{S}$. Lord, Muonium donor in rutile $\mathrm{TiO}_{2}$ and comparison with hydrogen, Phys. Rev. B 92, 081202 (2015).

[20] S. F. J. Cox, J. L. Gavartin, J. S. Lord, S. P. Cottrell, J. M. Gil, H. V. Alberto, J. Piroto Duarte, R. C. Vilão, N. Ayres 
de Campos, D. J. Keeble, E. A. Davis, M. Charlton, and D. P. van der Werf, Oxide muonics: II. Modelling the electrical activity of hydrogen in wide-gap and high-permittivity dielectrics, J. Phys.: Condens. Matter 18, 1079 (2006).

[21] R. B. L. Vieira, R. C. Vilão, H. V. Alberto, J. M. Gil, A. Weidinger, B. B. Baker, P. W. Mengyan, and R. L. Lichti, High-field study of muonium states in $\mathrm{HfO}_{2}$ and $\mathrm{ZrO}_{2}$, J. Phys.: Conf. Ser. 551, 012048 (2014).

[22] J. M. Gil, H. V. Alberto, R. C. Vilão, J. Piroto Duarte, N. Ayres de Campos, A. Weidinger, E. A. Davis, and S. F. J. Cox, Muonium states in $\mathrm{HgO}$, J. Phys.: Condens. Matter 13, L613 (2001).

[23] J. S. Lord, S. P. Cottrell, P. J. C. King, H. V. Alberto, N. Ayres de Campos, J. M. Gil, J. Piroto Duarte, R. C. Vilão, R. L. Lichti, S. K. L. Sjue, B. A. Bailey, A. Weidinger, E. A. Davis, and S. F. J. Cox, Probing the shallow-donor muonium wave function in $\mathrm{ZnO}$ and $\mathrm{CdS}$ via transferred hyperfine interactions, Phys. B: Condens. Matter 308-310, 920 (2001).

[24] E. Fortunato, P. Barquinha, and R. Martins, Oxide semiconductor thin-film transistors: A review of recent advances, Adv. Mater. 24, 2945 (2012).

[25] L. Petti, N. Münzenrieder, C. Vogt, H. Faber, L. Büthe, G. Cantarella, F. Bottacchi, T. D. Anthopoulos, and G. Tröster, Metal oxide semiconductor thin-film transistors for flexible electronics, Appl. Phys. Rev. 3, 021303 (2016).

[26] R. Gillen and J. Robertson, Electronic structure of lanthanide oxide high K gate oxides, Microelectron. Eng. 109, 72 (2013).

[27] A. G. Marinopoulos, Incorporation and migration of hydrogen in yttria-stabilized cubic zirconia: Insights from semilocal and hybrid-functional calculations, Phys. Rev. B 86, 155144 (2012).

[28] E. L. Silva, A. G. Marinopoulos, R. C. Vilão, R. B. L. Vieira, H. V. Alberto, J. Piroto Duarte, and J. M. Gil, Hydrogen impurity in yttria: Ab initio and $\mu$ SR perspectives, Phys. Rev. B 85, 165211 (2012)

[29] A. G. Marinopoulos, First-principles study of hydrogen configurations at the core of a high-angle grain boundary in cubic yttria-stabilized zirconia, J. Phys.: Condens. Matter 26, 025502 (2014).

[30] A. G. Marinopoulos, Protons in cubic yttria-stabilized zirconia: Binding sites and migration pathways, Solid State Ionics 315, 116 (2017).

[31] A. G. Marinopoulos, R. C. Vilão, H. V. Vieira, H. V. Alberto, J. M. Gil, M. V. Yakushev, R. Scheuermann, and T. Goko, Defect levels and hyperfine constants of hydrogen in beryllium oxide from hybrid-functional calculations and muonium spectroscopy, Philos. Mag. 97, 2108 (2017).

[32] R. C. Vilão, H. V. Alberto, J. P. Duarte, J. M. Gil, A. Weidinger, N. Ayres de Campos, R. L. Lichti, K. H. Chow, and S. F. J. Cox, Muonium spectroscopy in ZnSe: Metastability and conversion, Phys. Rev. B 72, 235203 (2005).
[33] R. C. Vilão, J. M. Gil, A. Weidinger, H. V. Alberto, J. Piroto Duarte, N. Ayres de Campos, R. L. Lichti, K. H. Chow, S. P. Cottrell, and S. F. J. Cox, Acceptor level of interstitial muonium in ZnSe and ZnS, Phys. Rev. B 77, 235212 (2008).

[34] G. Azimi, R. Dhiman, H.-M. Kwon, A. T. Paxson, and K. K. Varanasi, Hydrophobicity of rare-earth oxide ceramics, Nat. Mater. 12, 315 (2013).

[35] C. Kränkel, Rare-earth-doped sesquioxides for diode-pumped high-power lasers in the 1-, 2-, and 3- $\mu \mathrm{m}$ spectral range, IEEE J. Sel. Top. Quantum Electron. 21, 250 (2015).

[36] E. Bonera, G. Scarel, M. Fanciulli, P. Delugas, and V. Fiorentini, Dielectric Properties of High- $\kappa$ Oxides: Theory and Experiment for $\mathrm{Lu}_{2} \mathrm{O}_{3}$, Phys. Rev. Lett. 94, 027602 (2005).

[37] E. Heuer, G. Saraceno, M. Beil, P. Huber, and V. Kränkel, Efficient OPSL-pumped mode-locked $\mathrm{Yb}: \mathrm{Lu}_{2} \mathrm{O}_{3}$ laser with $67 \%$ optical-to-optical efficiency, Sci. Rep. 6, 19090 (2016).

[38] N.-L. Wang, X.-Y. Zhang, and P.-H. Wang, Fabrication and spectroscopic characterization of $\mathrm{Er}^{3+}: \mathrm{Lu}_{2} \mathrm{O}_{3}$ transparent ceramics, Mater. Lett. 94, 5 (2013).

[39] E. L. da Silva, A. G. Marinopoulos, R. B. L. Vieira, R. C. Vilão, H. V. Alberto, J. M. Gil, R. L. Lichti, P. W. Mengyan, and B. B. Baker, Electronic structure of interstitial hydrogen in lutetium oxide from DFT $+U$ calculations and comparison study with $\mu$ SR spectroscopy, Phys. Rev. B 94, 014104 (2016).

[40] More information at the webpage of the Centre for Molecular and Materials Science at TRIUMF: http://musr.ca/.

[41] More information at the webpage of the Muon Group at the ISIS Facility: https://www.isis.stfc.ac.uk/Pages/Muons.aspx.

[42] A. Meftah, F. Brisard, J. M. Costantini, E. Dooryhee, M. HageAli, M. Hervieu, J. P. Stoquert, F. Studer, and M. Toulemonde, Track formation in $\mathrm{SiO}_{2}$ quartz and the thermal-spike mechanism, Phys. Rev. B 49, 12457 (1994).

[43] H. B. Schlegel, Exploring potential energy surfaces for chemical reactions: An overview of some practical methods, J. Comput. Chem. 24, 1514 (2003).

[44] L. Gordon, H. Abu-Farsakh, A. Janotti, and C. G. Van de Walle, Hydrogen bonds in $\mathrm{Al}_{2} \mathrm{O}_{3}$ as dissipative two-level systems in superconducting qubits, Sci. Rep. 4, 7590 (2014).

[45] D. C. Walker, S. Karolczak, H. A. Gillis, and G. B. Porter, Hot model of muonium formation in liquids, Can. J. Chem. 81, 199 (2003).

[46] R. Berman, The thermal conductivity of dielectric solids at low temperatures, Adv. Phys. 2, 103 (1953).

[47] A. L. Woodcraft and A. Gray, A low temperature thermal conductivity database, in 13th International Workshop on Low Temperature Detectors (LTD'13), edited by B. Young, B. Cabrera, and A. Miller, AIP Conf. Proc. No. 1185 (AIP, New York, 2009), p. 681. 